\title{
Exciton dispersion from first principles
}

\author{
Matteo Gatti ${ }^{1,2,3,4}$ and Francesco Sottile ${ }^{1,2}$ \\ ${ }^{1}$ Laboratoire des Solides Irradiés, École Polytechnique, CNRS-CEA/DSM, F-91128 Palaiseau, France \\ ${ }^{2}$ European Theoretical Spectroscopy Facility (ETSF) \\ ${ }^{3}$ Nano-Bio Spectroscopy Group, Departamento Física de Materiales, Universidad del País Vasco, Centro de Física de Materiales \\ CSIC-UPVIEHU-MPC and DIPC, Avenida Tolosa 72, E-20018 San Sebastián, Spain \\ ${ }^{4}$ Synchrotron SOLEIL, L'Orme des Merisier, BP 48 Saint-Aubin, 91192 Gif sur Yvette, France \\ (Received 27 May 2013; revised manuscript received 19 September 2013; published 11 October 2013)
}

\begin{abstract}
We present a scheme to calculate exciton dispersions in real materials that is based on the first-principles many-body Bethe-Salpeter equation. We assess its high level of accuracy by comparing our results for LiF with recent inelastic x-ray scattering experimental data on a wide range of energy and momentum transfer. We show its great analysis power by investigating the role of the different electron-hole interactions that determine the exciton band structure and the peculiar "exciton revival" at large momentum transfer. Our calculations for solid argon are a prediction and a suggestion for future experiments. These results demonstrate that the first-principles Bethe-Salpeter equation is able to describe the dispersion of localized and delocalized excitons on equal footing and represent a key step for the ab initio study of the exciton mobility.
\end{abstract}

DOI: 10.1103/PhysRevB.88.155113

PACS number(s): 71.35.-y, 77.22.-d, 79.20.Uv

\section{INTRODUCTION}

The study of elementary excitations is one of the most powerful ways to understand, predict, and tune the properties of materials. In particular, excitons, i.e., collective lowenergy excitations involving the creation of electron-hole (e-h) pairs, are of paramount importance in elucidating the role of light absorption in, for example, the photovoltaic or photocatalytic process. ${ }^{1}$ Traditionally, excitons have been modeled following basic approximations, namely, the Frenkel and Wannier exciton models, which describe the two limiting cases of tightly and weakly bound e-h pairs, respectively. ${ }^{2,3}$ In most cases, however, interesting situations are intermediate between these two limits and model descriptions rapidly become very involved..$^{2-4}$ In the last couple of decades, instead, great advances have been obtained in the description of excitons thanks to many-body perturbation theory (MBPT) ${ }^{5}$ In particular, the Bethe-Salpeter equation (BSE) ${ }^{6}$ which is an effective two-particle equation for the polarizability, can nowadays be solved in an $a b$ initio framework ${ }^{7-9}$ allowing one to obtain absorption spectra in excellent agreement with experiments, to perform accurate analysis, and to successfully predict experimental results. ${ }^{10,11}$

Optical absorption spectroscopy probes the longwavelength limit $\mathbf{q} \rightarrow 0$. Thus, modern theoretical approaches for excitons have primarily focused on this $\mathbf{q} \rightarrow 0$ limit. However, excitons can carry a finite momentum $\mathbf{q}$ and form a band structure as a function of $\mathbf{q}$. The study of excitons at finite $\mathbf{q}$ and the determination of their band structure are interesting not only from an academic perspective. Indeed, for both weakly and tightly bound excitons, their $\mathbf{q}$ dispersion determines the way the e-h pairs propagate inside the crystal and transport their excitation energy. This property is of crucial relevance for all applications involving light harvesting, ${ }^{12,13}$ and also provides fundamental knowledge about exciton mobility and migration. ${ }^{14}$

The recent progress of scattering experiments such as electron energy-loss spectroscopy (EELS) or inelastic x-ray scattering (IXS), both in its resonant (RIXS) and nonresonant (NIXS) versions, makes it possible to probe electronic exci- tations at finite, and even large, momentum transfer $\mathbf{q}$ with great accuracy. ${ }^{15,16}$ Increasing $\mathbf{q}$ corresponds with a reduction in the probed length scale in real space. In fact, IXS and EELS are unique tools to measure electronic excitations that are dipole forbidden and thus not visible in optics. Typical examples are intra-atomic $d$ - $d$ excitations in strongly correlated transition-metal oxides ${ }^{17-19}$ or longitudinal $p$-like excitons in $s p$ cubic crystals. ${ }^{2,3,9}$ In addition, the new electron microscopes are able today to reach very high spatial resolution (well under $1 \mathrm{~nm}$ ) for the loss spectroscopy, ${ }^{20-22}$ which, in order to be investigated from the theoretical point of view, requires the knowledge of the dielectric function not at just $\mathbf{q}=0$, but at many q's, in order to Fourier transform back in real space. Concerning theory, however, ab initio simulations lie a long way behind experiments. Besides a few pioneering examples dealing with a small $\mathbf{q}$ range or core excitations, ${ }^{23-31}$ excitons in the range of large momentum transfers remain largely unexplored by first-principles approaches.

The present work closes the gap with experiment. The extension of the Bethe-Salpeter equation to finite momentum transfers makes it possible to describe and understand the exciton dispersion well beyond the first Brillouin zone. In order to illustrate the accuracy and the great power of analysis of the BSE, we have considered two prototypical materials displaying bound excitons: lithium fluoride, for which recent high-quality experiments are available, ${ }^{23,32-34}$ and solid argon, for which our spectra represent a prediction for future measurements. We show that, beyond model approaches, the ab initio BSE is able to simultaneously deal with strongly and weakly bound excitons, in a range of $\mathbf{q}$ vectors well beyond the optical limit. Moreover, the BSE formalism allows us to understand in a simple manner the exciton dispersion in terms of competing effects deriving from the electron band dispersion and the e-h interactions. The agreement with experiments in $\mathrm{LiF}$ is very good across the whole momentum-energy range, including the peculiar "exciton revival" at large $\mathbf{q}$, which we explain as a genuine many-body effect. In summary, these results illustrate the accuracy and the power of the BSE to study the exciton dispersion in a wide variety of materials, as well as to support 
and guide new experiments. The article is organized as follows: in Sec. II, we present the formula involved in the BSE (with finite momentum transfer) and, in Sec. III, the computational details; Sec. IV shows results and analysis for LiF, while Sec. V presents predictions for solid argon.

\section{BETHE-SALPETER EQUATION AT FINITE MOMENTUM TRANSFER}

The Bethe-Salpeter equation (BSE), based on the GW approximation (GWA) of the self-energy, reads $^{5}$

$$
\begin{aligned}
& L(1,2,3,4) \\
& =L_{0}(1,2,3,4)+\int d 5678 L_{0}(1,2,5,6)[v(5,7) \delta(5,6) \delta(7,8) \\
& \quad-W(5,6) \delta(5,7) \delta(6,8)] L(7,8,3,4),
\end{aligned}
$$

where (1) is a shorthand notation for position, time, and $\operatorname{spin}\left(\mathbf{r}_{1}, t_{1}, \sigma_{1}\right), L$ is the two-particle correlation function, $L_{0}$ is its independent-particle version, $v$ is the bare Coulomb interaction, and $W$ is its statically screened version calculated in the random-phase approximation.

The Dyson-like Eq. (1) can be reformulated ${ }^{10,11}$ as an eigenvalue problem by introducing the excitonic Hamiltonian $H_{\text {exc }}$ written on a basis of electron-hole transitions ${ }^{35} t$ : $\left(n_{1} \mathbf{k}_{1}\right) \rightarrow\left(n_{2} \mathbf{k}_{2}\right)$. According to the band index $n$ being an occupied state $(v)$ or an occupied state $(c)$, these are classified as resonant transitions, $\left(v, \mathbf{k}-\mathbf{q}_{\mathbf{r}}\right) \rightarrow(c, \mathbf{k})$, or antiresonant transitions, $(c, \mathbf{k}) \rightarrow\left(v, \mathbf{k}+\mathbf{q}_{\mathbf{r}}\right)$, where the momentum transfer $\mathbf{q}_{\mathbf{r}}$ belongs to the first Brillouin zone. The matrix elements are then

$$
\left\langle t\left|H_{\mathrm{exc}}\right| t^{\prime}\right\rangle=E_{t} \delta_{t, t^{\prime}}+\left\langle t|v-W| t^{\prime}\right\rangle,
$$

where $E_{t}$ is the energy associated to the transition (calculated in GWA), and

$$
\begin{aligned}
\left\langle t|v| t^{\prime}\right\rangle & =\left\langle n_{1} \mathbf{k}_{1} n_{2} \mathbf{k}_{2}|v| n_{1}^{\prime} \mathbf{k}_{1}^{\prime} n_{2}^{\prime} \mathbf{k}_{2}^{\prime}\right\rangle \\
& =2 \delta_{M} \int d \mathbf{r} d \mathbf{r}^{\prime} \phi_{n_{2} \mathbf{k}_{2}}^{*}(\mathbf{r}) \phi_{n_{1} \mathbf{k}_{1}}(\mathbf{r}) v\left(\mathbf{r}, \mathbf{r}^{\prime}\right) \phi_{n_{2}^{\prime} \mathbf{k}_{2}^{\prime}}\left(\mathbf{r}^{\prime}\right) \phi_{n_{1}^{\prime} \mathbf{k}_{1}^{\prime}}^{*}\left(\mathbf{r}^{\prime}\right) \\
\left\langle t|W| t^{\prime}\right\rangle & =\left\langle n_{1} \mathbf{k}_{1} n_{2} \mathbf{k}_{2}|W| n_{1}^{\prime} \mathbf{k}_{1}^{\prime} n_{2}^{\prime} \mathbf{k}_{2}^{\prime}\right\rangle \\
& =\int d \mathbf{r} d \mathbf{r}^{\prime} \phi_{n_{2} \mathbf{k}_{2}}^{*}(\mathbf{r}) \phi_{n_{2}^{\prime} \mathbf{k}_{2}^{\prime}}(\mathbf{r}) W\left(\mathbf{r}, \mathbf{r}^{\prime}\right) \phi_{n_{1} \mathbf{k}_{1}}\left(\mathbf{r}^{\prime}\right) \phi_{n_{1}^{\prime} \mathbf{k}_{1}^{\prime}}^{*}\left(\mathbf{r}^{\prime}\right)
\end{aligned}
$$

are the repulsive exchange electron-hole (e-h) interaction and the direct e-h attractive interaction, respectively. In Eq. (3), $\delta_{M}=1$ for the singlet channel and $\delta_{M}=0$ for the triplet. In this basis, $H_{\text {exc }}$ takes a block matrix form,

$$
H_{\mathrm{exc}}=\left(\begin{array}{cc}
R & K^{R, A} \\
K^{A, R} & A
\end{array}\right) .
$$

When the off-diagonal coupling terms $K$ are set to 0 , the Tamm-Dancoff approximation (TDA) is retrieved.

In the long-wavelength limit $\mathbf{q}_{\mathbf{r}} \rightarrow 0, A=-R^{*}$ and $K^{A, R}=-\left[K^{R, A}\right]^{*}$. The diagonal blocks $A$ and $R$ are Hermitian and the coupling blocks $K$ are symmetric. For a generic momentum transfer $\mathbf{q}_{\mathbf{r}} \neq 0$, the antiresonant block $A$ can no longer be obtained from the resonant block $R\left(A \neq-R^{*}\right)$ and the coupling terms are no longer symmetric. This doubles the computational cost of calculating $H_{\text {exc }}$.

From the solution of the eigenvalue problem,

$$
H_{\mathrm{exc}}\left(\mathbf{q}_{\mathbf{r}}\right) A_{\lambda}\left(\mathbf{q}_{\mathbf{r}}\right)=E_{\lambda}\left(\mathbf{q}_{\mathbf{r}}\right) A_{\lambda}\left(\mathbf{q}_{\mathbf{r}}\right),
$$

where we have made explicit the dependence on the momentum transfer $\mathbf{q}_{\mathbf{r}}$, one can obtain the inverse macroscopic dielectric function $\epsilon_{M}^{-1}$. In the TDA, one has

$$
\epsilon_{M}^{-1}(\mathbf{q}, \omega)=1+\frac{8 \pi}{q^{2}} \sum_{\lambda} \frac{\left|\sum_{t} A_{\lambda}^{t}\left(\mathbf{q}_{\mathbf{r}}\right) \tilde{\rho}^{t}(\mathbf{q})\right|^{2}}{\omega-E_{\lambda}\left(\mathbf{q}_{\mathbf{r}}\right)+i \eta},
$$

where $\mathbf{q}=\mathbf{q}_{\mathbf{r}}+\mathbf{G}_{0}$ is the desired momentum transfer, $\mathbf{G}_{0}$ is a reciprocal lattice vector, and $\tilde{\rho}_{t}(\mathbf{q})=\left\langle\phi_{v \mathbf{k}-\mathbf{q}_{\mathbf{r}}}\left|e^{-i \mathbf{q r}}\right| \phi_{c \mathbf{k}}\right\rangle$ is the oscillator strength. The loss function $-\operatorname{Im} \epsilon_{\mathrm{M}}^{-1}$ can be compared with EELS or IXS spectra.

Alternatively, if the Coulomb interaction $v$ in the BSE is replaced by a modified interaction $\bar{v}$ that in the reciprocal space is equal to $v$ for all the $\mathbf{G}$ components but $\mathbf{G}_{0}$ for which $\bar{v}\left(\mathbf{G}_{0}\right)=0$, then from the solution of the excitonic eigenvalue problem, one obtains the macroscopic dielectric function $\epsilon_{M}$ :

$$
\epsilon_{M}(\mathbf{q}, \omega)=1-\frac{8 \pi}{q^{2}} \sum_{\lambda} \frac{\left|\sum_{t} A_{\lambda}^{t}\left(\mathbf{q}_{\mathbf{r}}\right) \tilde{\rho}^{t}(\mathbf{q})\right|^{2}}{\omega-E_{\lambda}\left(\mathbf{q}_{\mathbf{r}}\right)+i \eta} .
$$

For simplicity, the notation is not modified with respect to Eq. (7), but here it is understood that $A_{\lambda}$ and $E_{\lambda}$ are the solution of the BSE with a modified Coulomb interaction $\bar{v}$. The long-range component of $v$ that is omitted in $\bar{v}$ is responsible for the splitting at $\mathbf{q}=0$ between longitudinal and transverse excitons in $s p$ cubic crystals. ${ }^{2,3,9}$ In the $\mathbf{q} \rightarrow 0$ limit, $\operatorname{Im} \epsilon_{\mathrm{M}}$ calculated from Eq. (8) gives the absorption spectrum (in which only transverse excitons are visible). At large momentum transfer, the long-range component of $v$ becomes less and less important, since increasing $\mathbf{q}$ means probing a shorter length scale in real space. Moreover, at large momentum transfer,

$$
\begin{aligned}
-\operatorname{Im} \epsilon_{M}^{-1}(\mathbf{q}, \omega) & =\frac{\operatorname{Im} \epsilon_{\mathrm{M}}(\mathbf{q}, \omega)}{\left[\operatorname{Re} \epsilon_{\mathrm{M}}(\mathbf{q}, \omega)\right]^{2}+\left[\operatorname{Im} \epsilon_{\mathrm{M}}(\mathbf{q}, \omega)\right]^{2}} \\
& \rightarrow \operatorname{Im} \epsilon_{\mathrm{M}}(\mathbf{q}, \omega),
\end{aligned}
$$

since $\operatorname{Re} \epsilon_{M} \rightarrow 1$ and $\operatorname{Im} \epsilon_{M}$ is small compared to 1 .

Besides the spectrum, the solution of the BSE allows a straightforward analysis, for instance by visualizing the exciton wave function of the e-h pair in real space, e.g., for $\mathbf{q}=0$ :

$$
\Psi_{\lambda}\left(\mathbf{r}_{h}, \mathbf{r}_{e}\right)=\sum_{t} A_{\lambda}^{t} \phi_{v \mathbf{k}}\left(\mathbf{r}_{h}\right) \phi_{c \mathbf{k}}\left(\mathbf{r}_{e}\right)
$$

\section{A. Comparison with the independent-particle picture}

In order to have a clear idea about how the excitonic effects (or the many-body effects) are taken into account in a theory that uses an independent-particle basis [such as densityfunctional theory (DFT) Kohn-Sham], it is useful to write the analogous equation of Eq. (8) for an independent-particle system:

$$
\epsilon_{M}(\mathbf{q}, \omega)=1-\frac{8 \pi}{q^{2}} \sum_{t} \frac{\left|\tilde{\rho}^{t}(\mathbf{q})\right|^{2}}{\omega-\epsilon_{t}+i \eta}
$$


The similarity is striking: in the latter formula, the (independent) transitions are summed up in a simple way, and their contribution to the final spectrum can only be in the energy range of the energy transition. In Eq. (8), instead, the transitions do not add up in a simple way, but they are mixed all together, via the eigenvectors of the excitonic Hamiltonian $A_{\lambda}^{t}\left(\mathbf{q}_{\mathbf{r}}\right)$, giving contribution to every exciton (every $\lambda$ ).

\section{COMPUTATIONAL DETAILS}

We have calculated the ground state of $\mathrm{LiF}$ and Ar using density-functional theory ${ }^{36}$ within the local-density approximation (LDA), ${ }^{37}$ using norm-conserving Troulliers-Martins pseudopotentials $^{38}$ in a plane-wave approach. In LiF, the energy cutoff is 40 Hartree, and we use a $12 \times 12 \times 12$ $\Gamma$-centered k-point grid. In Ar, the cutoff is also 40 Hartree and a finer grid with 4000 inequivalent $\mathbf{k}$ points is needed. In $\mathrm{LiF}$, we have corrected the LDA band structure with a scissor operator of $6.05 \mathrm{eV}$ for the band gap, and stretching the valence-band energies by $15 \%$ and conduction bands by $2 \%$. These corrections bring the LDA band structure into agreement with photoemission. ${ }^{8,24}$ Analogously, the scissor correction is $6.6 \mathrm{eV}$ in $\mathrm{Ar}^{39}{ }^{39} \mathrm{BSE}$ spectra are converged with 20 bands for $\mathrm{LiF}$ and 10 bands for Ar. The TDA that is employed for those spectra (see Sec. II) has no influence on the bound excitons. The effect of the coupling is mainly visible for the plasmon peak and becomes more important at larger $\mathbf{q}$. In the analysis of the exciton band dispersion in Fig. 4 , a $4 \times 4 \times 4$ k-point grid is used with three occupied and one unoccupied bands.

\section{LITHIUM FLUORIDE}

NIXS measures the dynamic structure factor $S(\mathbf{q}, \omega)$ that is proportional to the loss function $-\operatorname{Im} \epsilon_{M}^{-1}(\mathbf{q}, \omega)$ (the latter can be equivalently obtained also by EELS $):{ }^{40} S(\mathbf{q}, \omega)=-q^{2} /$ $\left(4 \pi^{2} n\right) \operatorname{Im} \epsilon_{M}^{-1}(\mathbf{q}, \omega)$ ( $n$ is the average electron density). The inverse of macroscopic dielectric function $\epsilon_{M}^{-1}$ can be directly calculated from the BSE, as seen in Sec. II.

In Fig. 1, we compare our BSE calculations (right panel) with experiment ${ }^{32}$ (left panel) for the dynamic structure factor of $\mathrm{LiF}$ over a wide range of energies (vertical axis) and momentum transfers (horizontal axis) along the $\Gamma X$ direction. ${ }^{41}$ We observe (i) a tightly bound exciton, dispersing around $14 \mathrm{eV}$ (the quasiparticle band gap lies between 14.98 and $16.17 \mathrm{eV}$ ) with a modulation in intensity until $\sim 6 \Gamma X$, and (ii) the valence plasmon at $\sim 23 \mathrm{eV}$, which fades out with increasing q. The agreement between theory and experiment is very good, both for the main features and for the fine structures in the spectra, generalizing previous results at selected $\mathbf{q}<1.5 \Gamma X .^{23,24}$ Here we focus on the bound exciton, which is mainly formed by e-h pairs from the three $\mathrm{F} 2 p$ valence bands and the first $\mathrm{Li} 2 s$ conduction band. In the optical limit, the two degenerate transverse excitons are seen in the absorption spectrum..$^{8,9,42}$ The third longitudinal exciton is visible in IXS at finite $\mathbf{q}$ along $\Gamma X$. The diagonalization of the excitonic Hamiltonian can also be achieved along different q directions, giving rise to a full description of the exciton band structure, as we show in Fig. 2. Thus, BSE calculations can complement experiments and obtain the band dispersion even for the excitons that are not visible in the spectra, but can be important, e.g., as deexcitation channels. For example, this is the case of the transverse excitons along $\Gamma X$. In other less symmetric directions such as $\Gamma W$, we find that both longitudinal and transverse branches become visible at the same time, confirming previous expectations ${ }^{2}$ and very recent results. $^{33}$

Inspection of the exciton wave function $\Psi\left(\mathbf{r}_{e}, \mathbf{r}_{h}\right)$ [see Eq. (10)] at $\mathbf{q}=0$ reveals that, consistently with the large binding energy, each e-h pair is confined in a small volume of the crystal. We plot in Fig. 3 the wave function of the first exciton of $\mathrm{LiF}$ at $\mathbf{q}=0$. This is defined as the probability distribution for the electron when the hole has been placed in $\mathbf{r}_{h}$. We report here the singlet (confirming the picture obtained, e.g., in Refs. 9 and 42) and the triplet low-lying excitation. It is worth highlighting three points: (i) even though the exciton is centered on the fluorine atom where the hole is fixed, it is delocalized over several primitive cells; (ii) the electron distribution, in addition, is not only localized on the same fluorine atom, but also on the nearest- and second-nearestneighboring fluorine atoms, avoiding the lithium atoms, even though the empty $2 s$ state of lithium is available, which shows how the physical picture can be counterintuitive if we rely only
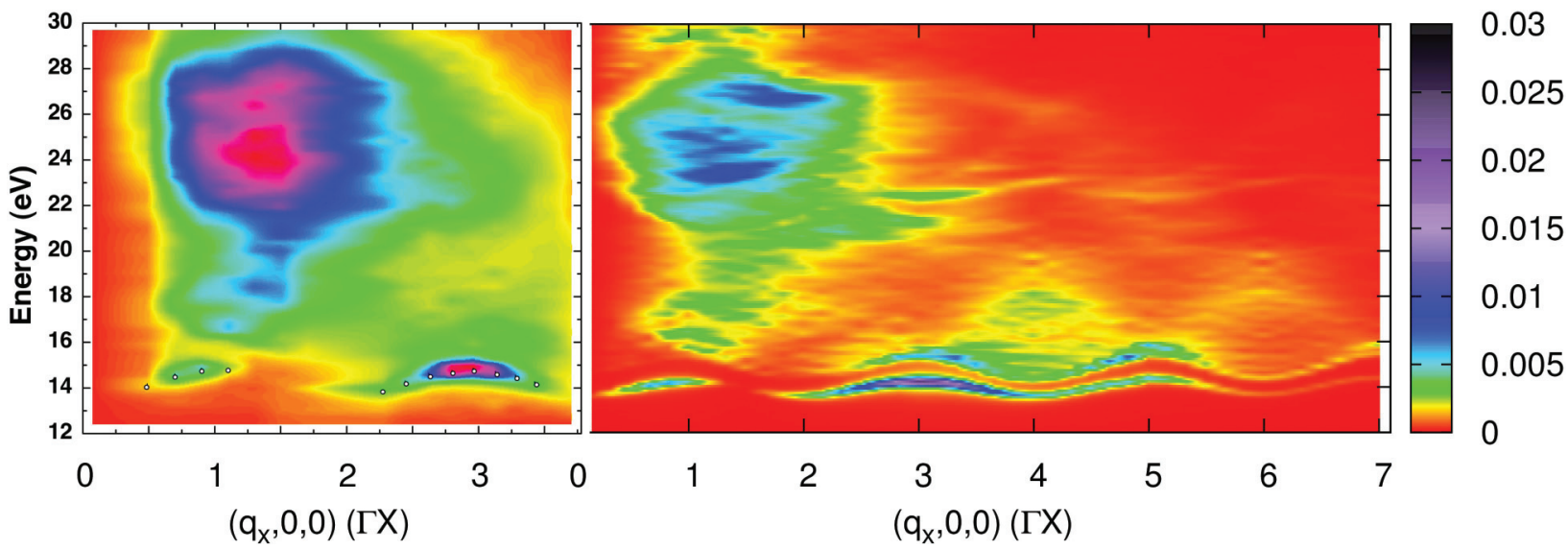

0.03

0.025

0.02

0.015

0.01

0.005

FIG. 1. (Color online) Dynamic structure factor of LiF as a function of energy (vertical axis) and momentum transfer $\mathbf{q}$ (horizontal axis) along $\Gamma X$ in units of $\Gamma X$. Left panel: Experimental data from Ref. 32. Right panel: Bethe-Salpeter calculations. The absolute scale for theoretical data is reported, but none is available for experimental data. 


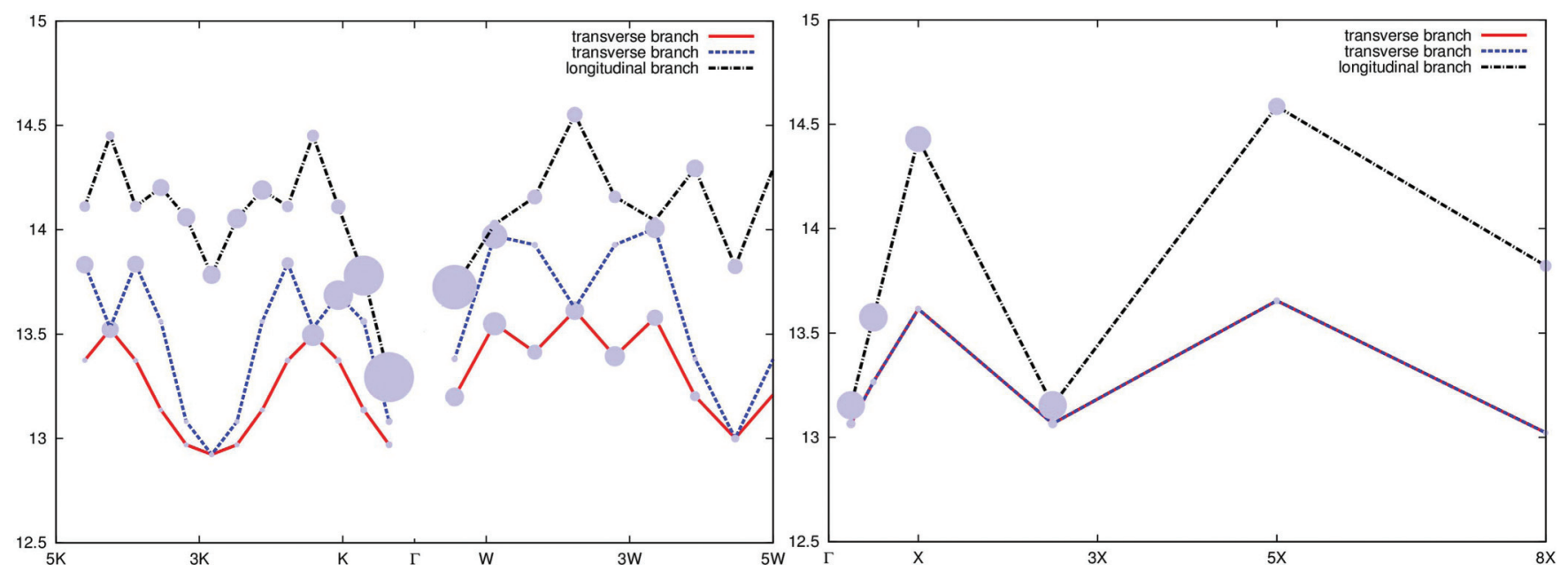

FIG. 2. (Color online) Exciton band structure of LiF. Three directions are investigated: $\Gamma X, \Gamma K$, and $\Gamma W$.

on a one-particle approximation; and (iii) the triplet exciton wave function shows a very similar picture as the singlet, with the electron distribution again localized on $\mathrm{F}$ atoms and not on $\mathrm{Li}$ atoms. This shows that the exchange electron-hole interaction is not qualitatively affecting the nature of the exciton.

In order to get a deeper understanding of the excitonic interactions at play in $\mathrm{LiF}$, we can have a critical look at the energy-momentum map of $S(\mathbf{q}, \omega)$ in Fig. 1 in the exciton region $(13<\omega<15 \mathrm{eV})$, and ask the following series of questions: (i) Why does the dynamical structure factor fade for high q? (ii) What is the origin of the exciton dispersion? (iii) Why does the intensity of the exciton increase with $\mathbf{q}$ up to $1 \Gamma X$, disappear, and come back stronger than before up to $3 \Gamma X$, to finally decay? It is easy to first answer question (i), for the decay of the independent-particle oscillator strengths $\tilde{\rho}^{t}(\mathbf{q})$ with increasing $\mathbf{q}$.

Question (ii) requires a deeper analysis, which is summarized in Fig. 4(a). The top-left panel represents the full ab initio calculation within a minimal model that includes $\mathrm{F}$ $2 p$ and $\mathrm{Li} 2 s$ bands; the top-right panel is a calculation in which we have artificially suppressed the band dispersion (same energy for every $\mathbf{k}$ ). The latter situation corresponds to the description that would be given by the simple Frenkel exciton model. Bottom panels are the equivalent triplet calculations, i.e., there is no e-h exchange interaction [ $v$ term in Eq. (2)]. Finally, in the bottom-right spectrum, the exciton dispersion is given only by e-h direct interaction $W$. Such dispersion, though small, precludes considering the overlap between wave functions localized on different atoms negligible in $\mathrm{LiF}^{43}$ The biggest contribution to the final exciton dispersion derives from the true electronic band dispersion [which gives $E_{\lambda}(\mathbf{q})$ a curvature that is opposite to the one obtained with the "flat bands" model], as can be seen by comparing the two top panels. This also implies that the necessary condition for simplified models to properly describe the exciton dispersion in $\mathrm{LiF}$ is to take into account (implicitly or explicitly) the electronic band dispersion. $^{4,32,33}$ It is interesting to compare also the triplet with the singlet, in order to directly evaluate the e-h exchange term, which has the effect of tempering the exciton dispersion, which otherwise would become too big. The final bandwidth of the exciton is $0.67 \mathrm{eV}$. Our ab initio calculations directly reproduce the experimental results and provide the tools for their analysis. It is worth noting that this analysis would be
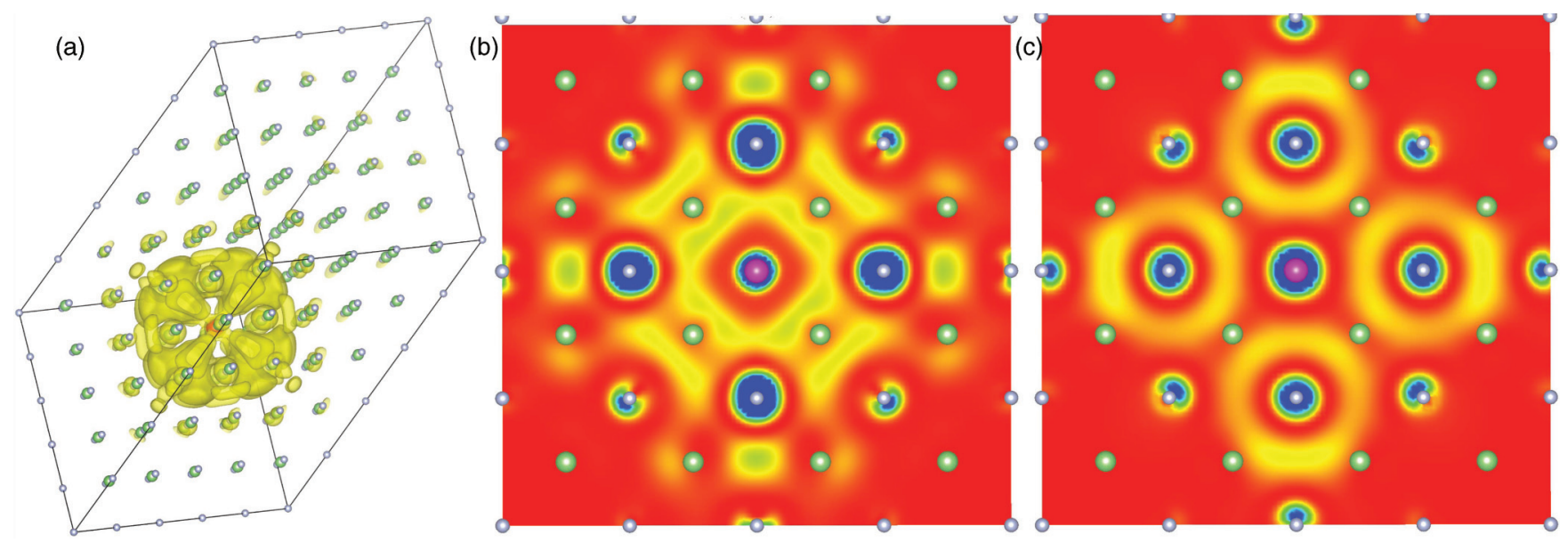

FIG. 3. (Color online) (a) Exciton wave function of LiF, when the hole is on top of the fluorine atom (violet in the center of the cell). (b) Cut along the [111] direction of the exciton wave function. (c) Same as (b), but for the triplet excitation. The physical picture is unchanged. 
(a)

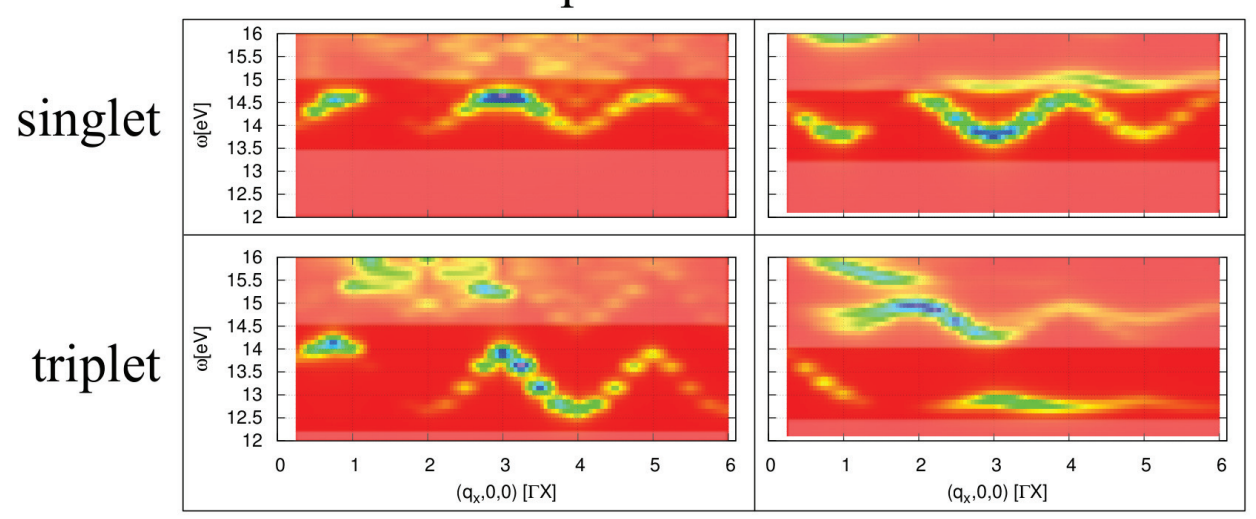

(b) Cumulative function

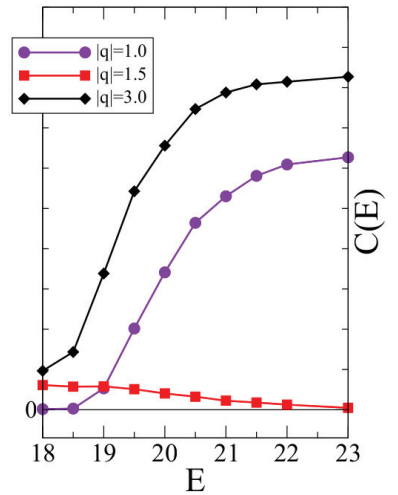

FIG. 4. (Color online) (a) Exciton dispersion in LiF in a minimal F $2 p$ and $\mathrm{Li} 2 s$ model with "flat bands" or with the full band dispersion for the singlet and the triplet. (b) Cumulative function $C_{\lambda}^{\mathbf{q}}(E)$ for the bound exciton in LiF as a function of the e-h transition energy $E$ (see text) for the intensity maxima at $\mathbf{q}=\Gamma X$ and $3 \Gamma X$, and for the minimum at $\mathbf{q}=1.5 \Gamma X$ (see Fig. 1).

very difficult by using simple models, such as Frenkel (that would hardly capture the band dispersion, which is the main cause of the exciton dispersion) or Wannier (that would impose the same parabolic dispersion to all excitons).

We can now tackle question (iii), which involves the important modulation of the exciton intensity (see Fig. 1). The exciton peak has a maximum at $\mathbf{q} \sim \Gamma X$; then it almost disappears before having a second and stronger maximum at $\mathbf{q} \sim 3 \Gamma X$ (beyond this point the intensity decreases until the exciton definitively disappears). In order to explain this "exciton revival" at large $\mathbf{q}$, we analyze the numerator of Eq. (7). The modulation in intensity for $\mathbf{q}<3 \Gamma X$ cannot be explained by considering separately $\tilde{\rho}^{t}(\mathbf{q})$ and the eigenvectors $A_{\lambda}^{t}(\mathbf{q})$. In fact, their behavior does not show a clear pattern as a function of q. Instead, we find that only by analyzing the $\tilde{\rho}^{t} A_{\lambda}^{t}(\mathbf{q})$ product as a whole is it possible to understand such an exciton revival. Indeed, the intensity of the exciton peak is determined by the constructive coherent superposition of the oscillator strengths from the different e-h configurations that form the excitonic state. To visualize explicitly the building up of the exciton as a function of the e-h transitions of energies $E_{t}$, in Fig. 4(b) we represent the cumulative function ${ }^{44}$

$$
C_{\lambda}^{\mathbf{q}}(E)=\int_{0}^{E} d \omega \sum_{t} A_{\lambda}^{t}(\mathbf{q}) \tilde{\rho}^{t}(\mathbf{q}) \delta\left(\omega-E_{t}\right)
$$

for three representative $\mathbf{q}=\Gamma X, 1.5 \Gamma X$, and $3 \Gamma X$. First of all, we realize that to quantitatively reproduce the intensity of the calculated spectrum, we have to take into account transitions spanning a wide energy range of at least $\sim 5 \mathrm{eV}$. Moreover, for the two maxima of spectral intensity at $\mathbf{q}=\Gamma X$ and $3 \Gamma X$, we see that different e-h transitions are always summing up constructively and, as a consequence, $C_{\lambda}^{\mathbf{q}}(E)$ is an increasing function of the energy $E$. Instead, for the minimum at $\mathbf{q}=1.5 \Gamma X$, the superposition of the different e-h configurations is constructive up to $E=18 \mathrm{eV}$, but then it becomes destructive for e-h transitions at higher energies, which leads to a reduction in the final intensity of the peak. This analysis illustrates once more the many-body character of a collective excitation such as an exciton, which cannot be captured by any independent-particle picture.

\section{ARGON}

In view of the very good agreement with experiment for $\mathrm{LiF}$, we now move to solid argon for which our calculations (see Sec. III) represent a prediction for future measurements. Argon is a textbook material that has been studied extensively in the past ${ }^{2,39,45-50}$ for its absorption spectrum that shows a hydrogenlike bound-exciton series in which the $n=1$ exciton has a strongly localized character and the higher excitons $n \geqslant 2$ are more delocalized..$^{51}$ The energy-momentum dispersion map represented in Fig. 5 fully discloses the richness of the excitation spectrum beyond what can be seen in the optical limit. First of all, we see that from the experimental point of view, one should preferably make the measurement at $\mathbf{q}=3 \Gamma X$, where the peaks are the most intense. At this momentum transfer, there are two prominent excitons at 13.8 and $15.4 \mathrm{eV}$, i.e., inside the band gap (which is marked by the white line in Fig. 5) and in the e-h excitation continuum,

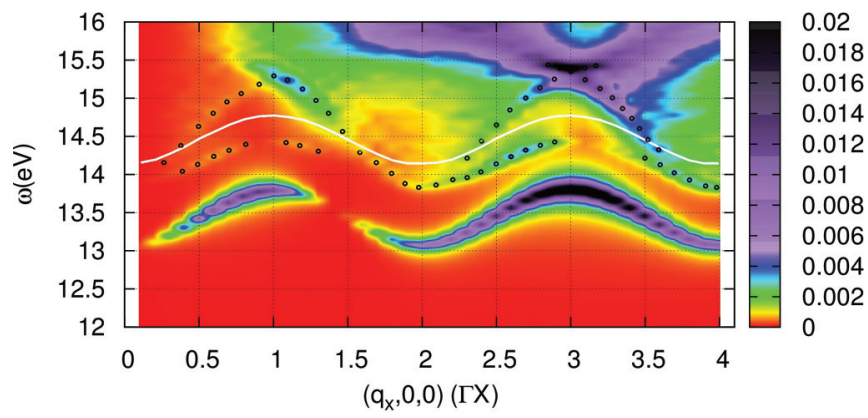

FIG. 5. (Color online) Dynamic structure factor of solid argon as a function of energy (vertical axis) and momentum transfer $\mathbf{q}$ (horizontal axis) along $\Gamma X$ in units of $\Gamma X$ : predictions from the Bethe-Salpeter equation. The white line is the quasiparticle direct band gap as a function of $\mathbf{q}$. The dots mark the dispersion of two excitons located between the two most prominent peaks. 
respectively. The bound exciton, which corresponds to most intense $n=1$ peak in the absorption spectrum, has a dispersion that recalls that of the lowest-energy exciton in $\mathrm{LiF}$, while the dispersion of the resonant exciton at higher energies has an opposite curvature and is more intense outside the first Brillouin zone. In the energy range between these two excitons, several other weaker features appear (some of them are dipole forbidden and hence not visible in the absorption spectrum). In Fig. 5, the most intense among them are marked by the small dots. We find that the various exciton branches have a large bandwidth (up to $1 \mathrm{eV}$ ) and they show different dispersions. This is in contrast to the simple Wannier model in which the exciton energy levels of the Rydberg series should all have the same dispersion. We thus see that the first-principles $\mathrm{BSE}$ is able to describe at the same time the dispersion of a large variety of excitons, as shown here in solid Ar, from the more localized $n=1$ exciton to the more delocalized resonant excitons. This would be very hard to achieve using a single model.

\section{CONCLUSIONS}

In conclusion, we have shown how the $a b$ initio BetheSalpeter equation is able to accurately describe the dispersion of plasmons and localized and delocalized excitons on equal footing. We have calculated the band structure of visible and dark excitons in $\mathrm{LiF}$ and analyzed its behavior in terms of com- peting electron-hole interactions. Our results for solid argon are a prediction for future experiments. This work opens the way for a large variety of applications, from the investigation of vertex corrections beyond the $\mathrm{GW}$ approximation for spectral properties to the study of dispersion of orbiton excitations in transition-metal oxides, ${ }^{52}$ and represents a fundamental step towards an ab initio analysis of exciton propagation in real materials. $^{13}$

\section{ACKNOWLEDGMENTS}

We thank Lucia Reining, Pina Romaniello, Pierluigi Cudazzo, Angel Rubio, and Simo Huotari for fruitful discussions, and Joanne Oates for critical reading. We thank Jean-Pascal Rueff and Peter Abbamonte for providing us with the corrected results from Ref. 32. We acknowledge financial support from Triangle de la Physique 2013-0013T IXST, the ERC Advanced Grant DYNamo (No. ERC-2010-AdG267374), Spanish Grants (No. 2010-21282-C02-01 and No. PIB2010US-00652), Grupos Consolidados UPV/EHU del Gobierno Vasco (Grant No. IT578-13) and European Commission projects CRONOS (Grant No. 280879-2 CRONOS CP-FP7). Computational time was granted by GENCI (Project No. 544) and by the CNANO-SIMULEE-2009 project. Ground-state calculations are carried out with the ABINIT package, ${ }^{53}$ while the dynamical structure factor, and all exciton analyses, are calculated with the EXC code. ${ }^{54}$
${ }^{1}$ A. Fujishima, X. Zhang, and D. A. Tryk, Surf. Sci. Rep. 63, 515 (2008).

${ }^{2}$ R. S. Knox, Theory of Excitons (Academic, New York, 1963).

${ }^{3}$ F. Bassani and G. P. Parravicini, Electronic States and Optical Transitions in Solids (Pergamon, New York, 1975).

${ }^{4}$ See, e.g., M. Altarelli and B. F. Bassani, J. Phys. C 4, L328 (1971).

${ }^{5}$ G. Strinati, Rivista del Nuovo Cimento 11(12), 1 (1988).

${ }^{6}$ W. Hanke and L. J. Sham, Phys. Rev. Lett. 43, 387 (1979).

${ }^{7}$ S. Albrecht, L. Reining, R. Del Sole, and G. Onida, Phys. Rev. Lett. 80, 4510 (1998).

${ }^{8}$ L. X. Benedict, E. L. Shirley, and R. B. Bohn, Phys. Rev. Lett. 80, 4514 (1998).

${ }^{9}$ M. Rohlfing and S. G. Louie, Phys. Rev. Lett. 81, 2312 (1998).

${ }^{10}$ M. Rohlfing and S. G. Louie, Phys. Rev. B 62, 4927 (2000).

${ }^{11}$ G. Onida, L. Reining, and A. Rubio, Rev. Mod. Phys. 74, 601 (2002).

${ }^{12}$ G. Trinkunas, O. Zerlauskiene, V. Urbonien, J. Chmeliov, A. Gall, B. Robert, and L. Valkunas, J. Phys. Chem. B 116, 5192 (2012).

${ }^{13}$ D. A. Wheeler and J. Z. Zhang, Adv. Mater. 25, 2878 (2013).

${ }^{14}$ R. M. Hochstrasser and J. D. Whiteman, J. Chem. Phys. 56, 5945 (1972).

${ }^{15}$ W. Schülke, Electron Dynamics by Inelastic X-ray Scattering (Oxford University Press, Oxford, UK, 2007).

${ }^{16}$ F. J. García de Abajo, Rev. Mod. Phys. 82, 209 (2010).

${ }^{17}$ B. C. Larson, J. Z. Tischler, W. Ku, C. C. Lee, O. D. Restrepo, A. G. Eguiluz, P. Zschack, and K. D. Finkelstein, Phys. Rev. Lett. 99, 026401 (2007).
${ }^{18}$ N. Hiraoka, H. Okamura, H. Ishii, I. Jarrige, K. D. Tsuei, and Y. Q. Cai, Eur. Phys. J. B 70, 157 (2009).

${ }^{19}$ F. Iori, F. Rodolakis, M. Gatti, L. Reining, M. Upton, Y. Shvyd'ko, J.-P. Rueff, and M. Marsi, Phys. Rev. B 86, 205132 (2012).

${ }^{20}$ S. Mazzucco, O. Stéphan, C. Colliex, I. Pastoriza-Santos, L. LizMarzan, F. J. García de Abajo, and M. Kociak, Eur. Phys. J. Appl. Phys. 54, 33512 (2011).

${ }^{21}$ A. Rivacoba, N. Zabala, and J. Aizpurua, Prog. Surf. Sci. 65, 1 (2000).

${ }^{22}$ F. J. García de Abajo and M. Kociak, Phys. Rev. Lett. 100, 106804 (2008).

${ }^{23}$ W. A. Caliebe, J. A. Soininen, E. L. Shirley, C.-C. Kao, and K. Hämäläinen, Phys. Rev. Lett. 84, 3907 (2000).

${ }^{24}$ J. A. Soininen and E. L. Shirley, Phys. Rev. B 61, 16423 (2000).

${ }^{25}$ S. Galambosi, J. A. Soininen, K. Hämäläinen, E. L. Shirley, and C.-C. Kao, Phys. Rev. B 64, 024102 (2001).

${ }^{26}$ A. Marini, R. Del Sole, and A. Rubio, Phys. Rev. Lett. 91, 256402 (2003).

${ }^{27}$ H. K. Mao, E. L. Shirley, Y. Ding, P. Eng, Y. Q. Cai, P. Chow, Y. Xiao, J. Shu, R. J. Hemley, C. Kao, and W. L. Mao, Phys. Rev. Lett. 105, 186404 (2010).

${ }^{28}$ C.-C. Lee, H. C. Hsueh, and W. Ku, Phys. Rev. B 82, 081106 (2010).

${ }^{29}$ S. Sharma, J. K. Dewhurst, A. Sanna, A. Rubio, and E. K. U. Gross, New J. Phys. 14, 053052 (2012).

${ }^{30}$ K. Hämäläinen, S. Galambosi, J. A. Soininen, E. L. Shirley, J.-P. Rueff, and A. Shukla, Phys. Rev. B 65, 155111 (2002).

${ }^{31}$ J. Vinson, J. J. Rehr, J. J. Kas, and E. L. Shirley, Phys. Rev. B 83, 115106 (2011). 
${ }^{32}$ P. Abbamonte, T. Graber, J. P. Reed, S. Smadici, C.-L. Yeh, A. Shukla, J.-P. Rueff, and W. Ku, Proc. Natl. Acad. Sci. USA 105, 12159 (2008).

${ }^{33}$ C.-C. Lee, X. M. Chen, C.-L. Yeh, H. C. Hsueh, P. Abbamonte, and W. Ku, arXiv:1205.4106v1.

${ }^{34}$ W. A. Caliebe, Ph.D. thesis, Kiel Universität, Germany, 1997, http://www.ieap.uni-kiel.de/solid/ag-press/r/pd/diss1.htm

${ }^{35}$ For simplicity, here we drop the spin index. $H_{\text {exc }}$ can be easily generalized to spin-polarized systems. ${ }^{10}$

${ }^{36}$ P. Hohenberg and W. Kohn, Phys. Rev. 136, B864 (1964).

${ }^{37}$ W. Kohn and L. J. Sham, Phys. Rev. 140, A1133 (1965).

${ }^{38}$ N. Troullier and J. L. Martins, Phys. Rev. B 43, 1993 (1991).

${ }^{39}$ F. Sottile, M. Marsili, V. Olevano, and L. Reining, Phys. Rev. B 76, 161103 (2007).

${ }^{40}$ Throughout the paper, atomic units are used if not mentioned otherwise.

${ }^{41}$ The original figure in Ref. 32 contains an error that has been corrected by shifting the energy axis by $1.0 \mathrm{eV}$. J.-P. Rueff and P. Abbamonte (private communication).

${ }^{42}$ P. Puschnig and C. Ambrosch-Draxl, Phys. Rev. B 66, 165105 (2002).

${ }^{43}$ P. Cudazzo, M. Gatti, and A. Rubio, Phys. Rev. B 86, 195307 (2012).

${ }^{44}$ L. Yang, J. Deslippe, C.-H. Park, M. L. Cohen, and S. G. Louie, Phys. Rev. Lett. 103, 186802 (2009).

${ }^{45}$ G. Baldini, Phys. Rev. 128, 1562 (1962).
${ }^{46}$ R. Haensel, G. Keitel, E. E. Koch, M. Skibowski, and P. Schreiber, Phys. Rev. Lett. 23, 1160 (1969).

${ }^{47}$ V. Saile, M. Skibowski, W. Steinmann, P. Gürtler, E. E. Koch, and A. Kozevnikov, Phys. Rev. Lett. 37, 305 (1976).

${ }^{48}$ W. Andreoni, M. Altarelli, and F. Bassani, Phys. Rev. B 11, 2352 (1975).

${ }^{49}$ S. Baroni, G. Grosso, L. Martinelli, and G. Pastori Parravicini, Phys. Rev. B 20, 1713 (1979).

${ }^{50}$ S. Galamic-Mulaomerovic and C. H. Patterson, Phys. Rev. B 72, 035127 (2005)

${ }^{51}$ Each exciton is additionally split into doublets for the effect of the spin-orbit coupling, which is neglected in the calculation.

${ }^{52}$ J. Schlappa, K. Wohlfeld, K. J. Zhou, M. Mourigal, M. W. Haverkort, V. N. Strocov, L. Hozoi, C. Monney, S. Nishimoto, S. Singh, A. Revcolevschi, J.-S. Caux, L. Patthey, H. M. Ronnow, J. van den Brink, and T. Schmitt, Nature (London) 485, 82 (2012).

${ }^{53}$ X. Gonze, B. Amadon, P.-M. Anglade, J.-M. Beuken, F. Bottin, P. Boulanger, F. Bruneval, D. Caliste, R. Caracas, M. Ct, T. Deutsch, L. Genovese, P. Ghosez, M. Giantomassi, S. Goedecker, D. Hamann, P. Hermet, F. Jollet, G. Jomard, S. Leroux, M. Mancini, S. Mazevet, M. Oliveira, G. Onida, Y. Pouillon, T. Rangel, G.-M. Rignanese, D. Sangalli, R. Shaltaf, M. Torrent, M. Verstraete, G. Zerah, and J. Zwanziger, Comput. Phys. Commun. 180, 2582 (2009).

${ }^{54}$ L. Reining, V. Olevano, F. Sottile, S. Albrecht, and G. Onida, The exc code (unpublished), http://www.bethe-salpeter.org/ 